\title{
Calidad de vida y satisfacción postquirúrgica en pacientes pediátricos operados de Pectus excavatum mediante técnica de Nuss
}

\author{
JORGE VELARDE G. ${ }^{1}$, PAULINA SILVA T. ${ }^{2}$, ALEJANDRO KRAL B. ${ }^{3}$, LUIS BOFILL CH. $^{4}$ \\ 1. Cirujano Infantil. Hospital Dr. Gustavo Fricke. Jefe de Cátedra de Cirugía Pediátrica. Universidad de Valparaíso. \\ 2. Pediatra. Universidad de Valparaiso. \\ 3. Residente de Medicina Interna. Universidad de Valparaiso. \\ 4. Residente de Pediatria. Universidad de Valparaiso.
}

\section{ABSTRACT \\ Quality of life and postsurgical satisfaction among pediatric patients operated on for Pectus excavatum using nuss procedure}

Pectus excatavum is the most common congenital deformity of the anterior wall of the chest. This condition is approached surgically for cosmetic and/or functional purposes. The Nuss procedure is a minimally-invasive procedure with excellent cosmetic outcomes and is less traumatic than other techniques. This study was conducted to determine pediatric patients and their parents' postoperative satisfaction and changes in quality of life after the Nuss technique. Patients and Method: A descriptive and single cohort study was performed subjecting patients and their parents to evaluate their quality of life and postoperative satisfaction using the Nuss and Krasopoulos questionnaires. The data was analyzed based on sample of $n$, median, interquartile ranges and t-student test, with $\mathrm{p}<0.05$, according to paired-sample Wilcoxon test. Results: 31 patients were operated on for Pectus excatavum; most of the patients required operation for aesthetics and functional reasons. All the answers to questions in both questionnaires, Nuss and Krasopoulos, regarding differences between pre surgery and post-surgery were statistically significant, with $\mathrm{p}<0.05$ using t-student values. Conclusion: The correction of Pectus excatavum using the Nuss procedure has shown a positive effect physically and biopsychosocially on patients to correct the deformity, resulting in improved patients' self-esteem and perception of their health.

(Key words: Pectus excatavum, quality of life, Nuss procedure, thoracic malformations).

Rev Chil Pediatr 2013; 84 (2): 166-176

\section{RESUMEN}

Antecedentes: El Pectus excatavum es la deformidad congénita más frecuente de la pared anterior del tórax. Esta condición es abordada quirúrgicamente por causas estéticas y/o funcionales. La técnica quirúrgica de

Recibido el 29 de abril de 2012, devuelto para corregir el 24 de julio de 2012, segunda versión el 3 de septiembre de 2012, aceptado para publicación el 06 de febrero de 2013.

Este trabajo cumple con los requisitos sobre consentimiento /asentimiento informado, comité de ética, financiamiento, estudios animales y sobre la ausencia de conflictos de intereses según corresponda.

Correspondencia a:

Jorge Velarde Gaggero

E-mail: jorgevelardeg@yahoo.es 
Nuss destaca por tener mejores resultados estéticos y menor grado de traumatismo y complicaciones en comparación con otras técnicas. El objetivo del presente estudio fue conocer el cambio en la calidad de vida y el grado de satisfacción post operatoria, en los pacientes pediátricos y sus padres, operados mediante técnica de Nuss. Pacientes y Método: Se realizó un estudio descriptivo, de cohorte única, sometiendo a los pacientes y a sus padres a evaluación de la calidad de vida y satisfacción post operatoria mediante el cuestionario de Nuss y Krasopoulos, siendo los datos analizados, en base al n de la muestra, mediante mediana, rangos intercuartiles y t-student, con $\mathrm{p}<0,05$, según prueba pareada de Wilcoxon. Resultados: Se registraron 31 pacientes operados de Pectus excatavum, siendo la gran mayoría de causa tanto estética como funcional. Tanto en el cuestionario de Nuss, como en el de Krasopoulos, todas las respuestas a preguntas sobre diferencias pre cirugía y post cirugía fueron estadísticamente significativas, con un t-student de $p<0,05$. Conclusión: La corrección del Pectus excatavum mediante técnica de Nuss ha mostrado un efecto positivo, tanto en la esfera física, como biopsicosocial de los pacientes al corregir su deformidad, lo que se ve claramente reflejado en una mejora de la autoestima y en la percepción de su estado de salud, por parte de los pacientes.

(Palabras clave: Pectus excatavum, Calidad de vida, Técnica de Nuss, Malformaciones torácicas).

Rev Chil Pediatr 2013; 84 (2): 166-176

\section{Introducción}

Se define Pectus excatavum como una deformidad congénita de la pared torácica anterior, que afecta con mayor frecuencia a los cartílagos costales inferiores, produciendo un hundimiento en la pared anterior del tórax, que habitualmente es asimétrico y de profundidad variable ${ }^{1,2}$. Es la deformidad congénita más frecuente de la pared anterior del tórax, alcanzando un 90 ó 92\% de estas. Con una incidencia de 1:700 a 1:1000 RN vivos, afecta principalmente a varones y a la raza blanca. Un 37\% tienen antecedentes familiares de la misma patología ${ }^{3}$. Descrito inicialmente por Bauhinus en 1504, solamente fue reconocido como entidad clínica en el siglo XVI.

Se desarrolla en los primeros años de la vida cuando los lactantes comienzan a deambular y a participar en juegos infantiles, llamando la atención de los padres por la posición corporal en bipedestación con el pecho hundido, abdomen prominente, tórax flexionado con hombros redondeados y proyectados hacia el frente, que obligan al infante a hiperextender el cuello y la cabeza ${ }^{4}$.

$\mathrm{Su}$ etiopatogenia se atribuye al crecimiento anormal de los cartílagos costales, lo cual provoca desviación posterior de la porción distal del cuerpo del esternón y la base del apéndice xifoides, cuyo extremo distal se proyecta hacia delante. La lesión puede ser simétrica o asimétrica, predominando en frecuencia la asimetría derecha, probablemente debido al desarrollo del pericardio fibroso y su fusión con el centro frénico tendinoso $\mathrm{O}^{3,4}$.

Morfológicamente se ha encontrado una alteración en la distribución y organización del colágeno tipo II, que provoca menor resistencia por parte del cartílago. Otros estudios han demostrado que hay una disminución del zinc y un aumento del calcio y magnesio a nivel de los cartílagos costales. Por otro lado se postula que hay una causa genética, debido a que en alrededor del $35 \%$ de los casos se encuentra una historia familiar ${ }^{3-5}$.

La deformidad aumenta de manera progresiva en relación al crecimiento del niño y se hace más evidente en el período de la adolescencia, siendo muy pocos los casos que experimentan regresión espontánea o mejoría parcial.

La decisión para abordarlo quirúrgicamente se basa en: 1) causas estéticas; 2) pacientes sintomáticos por su patología; 3 ) índice de Haller en la TAC > 3,25; 4) alteraciones cardiológicas y 5) alteraciones respiratorias ${ }^{6,7}$. Debido a la deformación estética que esta malformación produce, se ve afectada drásticamente la autoestima y el desarrollo psico-social de los pacientes.

Desde 1998 a la fecha, se ha popularizado la técnica de Nuss para la reparación de esta malformación ${ }^{15}$. Esta técnica consiste en la inserción de una barra, previamente curvada, la cual se introduce a través del espacio retroesternal (entre el pericardio y la cara posterior 
del esternón) y se rota de cóncavo a convexo, consiguiendo la corrección de la deformidad. Esta barra se introducía inicialmente a ciegas (con mayor riesgo de lesión cardíaca, al realizarse la disección retroesternal sin visualización directa); lo que motivó un cambio en la técnica, para realizar luego el procedimiento bajo visión videotoracoscópica. Su aplicación en pacientes pediátricos se explica gracias a que ellos tienen un tórax blando y maleable y rara vez presentan fracturas costales en los traumatismos. Esta cirugía es menos traumática que la técnica tradicional, con menores complicaciones, menor tiempo operatorio y con resultados estéticos satisfactorios para los pacientes.

$\mathrm{Si}$ bien existen indicadores tradicionales que reflejan el éxito de la cirugía, como son la presencia o no de complicaciones y/o la mejora de parámetros de laboratorio, también adquiere importancia el desarrollo de una medida que tenga en cuenta la opinión (percepción) del paciente sometido a la cirugía o cualquier otro procedimiento que tenga relación con la forma en que el sujeto percibe su estado de salud-enfermedad. La información que proporcionan estas nuevas medidas, permite identificar distintos estados de morbilidad y bienestar (o malestar), valorar el impacto de distintos tratamientos y de las intervenciones sanitarias, identificar las características sociodemográficas de la población en estudio y la forma en que repercute el estado de salud-enfermedad en el ámbito físico, psicológico y social. Todo esto nos lleva al concepto de Calidad de Vida Relacionada con la Salud, el cual en niños/as y adolescentes no sólo considera los aspectos físicos, psicológicos y sociales, sino también la habilidad de realizar actividades apropiadas para su edad ${ }^{6}$. La salud autopercibida se asocia de manera independiente a los diagnósticos clínicos y permite predecir la hospitalización y la utilización de los servicios de atención primaria, por lo tanto, se considera una variable relevante asociada con el gasto sanitario ${ }^{12,13}$.

El objetivo del presente estudio fue conocer el cambio en la calidad de vida y el grado de satisfacción, tanto de los pacientes pediátricos, como de sus padres, operados mediante técnica de Nuss en nuestro centro.

\section{Pacientes y Método}

Estudio descriptivo, de cohorte única, realizado durante el mes de Julio de 2010, en las dependencias del Servicio de Cirugía Pediátrica del Hospital Dr. Gustavo Fricke de Viña del Mar.

Para este estudio se seleccionaron todos los pacientes operados de Pectus excatavum mediante técnica de Nuss entre noviembre de 2004 y abril de 2010 .

Se incluyeron las siguientes variables epidemiológicas: sexo, edad actual, edad a la cirugía, tiempo trascurrido, fecha de la cirugía, lugar de residencia (o destino en caso de pertenecer a otro centro asistencial), causal de la cirugía (se consideró como causa estética o por motivos orgánicos).

Se evaluó el índice de Haller preoperatorio, que establece la relación entre el diámetro transverso y antero posterior del tórax en la zona de mayor depresión esternal, se considera normal hasta 3,25 (figura 1).

Se revisó además la evaluación preoperatoria cardiológica, la espirometría, el tiempo operatorio, los días de estadía en UCIP, los requerimientos de analgesia, los días de estadía hospitalaria, la cantidad de barras/estabilizadores y tipo de barra utilizada y las complicaciones post quirúrgicas (Anexo 1. Variables epidemiológicas a analizar).

Para evaluar la calidad de vida post cirugía y satisfacción de esta se utilizó el cuestionario de Nuss para pacientes pediátricos ${ }^{14} \mathrm{el}$ cual

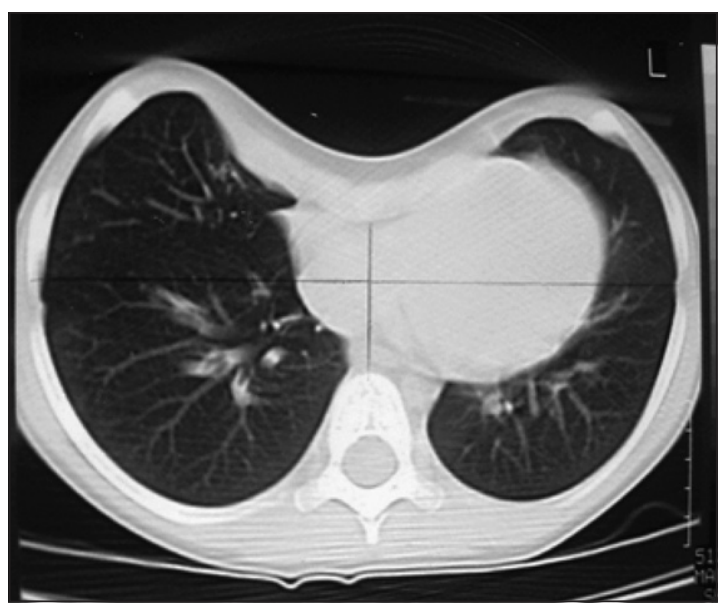

Figura 1. TAC de tórax. Índice de Haller. 

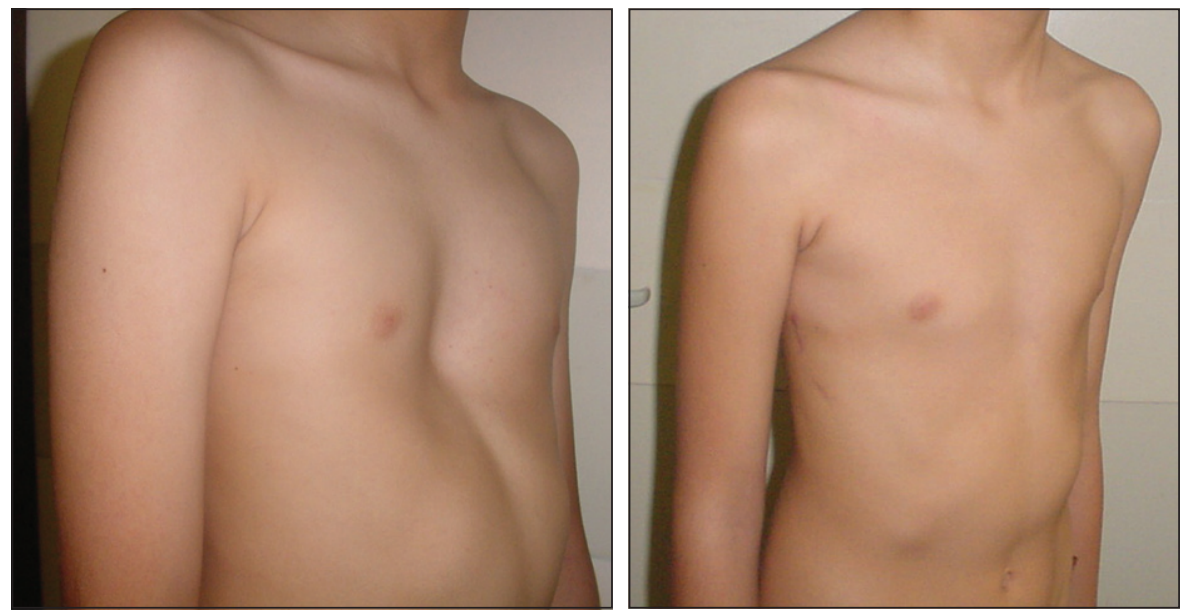

Figura 2. a) paciente de 7 años de edad foto preoperatoria; b) postoperado 6 meses después (con autorización).

fue aplicado a una población pediátrica (los pacientes y sus padres) y utilizado para determinar el efecto que este procedimiento tenía en el funcionamiento físico y psicosocial. Este consta de 12 preguntas, cada una de ellas se divide en dos partes, una pre cirugía y otra post cirugía, a su vez cada una tiene 4 posibilidades de respuestas las cuales van desde: muy feliz hasta muy infeliz. (Anexo 2: cuestionario de Nuss).

Además incluimos el cuestionario creado por Krasopoulos ${ }^{3}$, el cual permite evaluar el grado de satisfacción que tienen los pacientes, con el procedimiento al cual fueron sometidos. Este consta de 16 preguntas, evaluando el impacto social, estético y físico que ha acarreado la cirugía, en los niños menores de 10 años se les pidió a los padres que contesten las preguntas y a los mayores de esa edad, a ellos que las respondan. (Anexo 3: Cuestionario de satisfacción post quirúrgica).

Las encuestas de Nuss y Krasopoulos se encuentran previamente validadas ${ }^{16}$. Ambos cuestionarios fueron administrados vía telefónica al padre y/o menor intervenido, por alguno de los autores, para lo cual se recurrió a los registros telefónicos del servicio de Cirugía Pediátrica.

Posteriormente, los datos fueron ingresados a una planilla de Microsoft excel y analizados. Para describir las variables cuantitativas se describió mediante proporciones. Se usó prueba de Shapiro Wilk para evaluar distribución normal en las variables continuas, describiéndose si tenían distribución paramétrica mediante medias y desviaciones estándar y con mediana y rangos intercuartiles si tenían distribución no paramétrica. Los cruces entre variables cualitativas se hicieron con prueba de $\chi^{2}$ (si eran dicotómicas con prueba exacta de Fischer). Los cruces entre variables cualitativas y cuantitativas según prueba de Mann Whitney y se usó prueba pareada de Wilcoxon para evaluar los resultados pre y post test de la encuesta. Los cruces entre variables cuantitativas se evaluaron según prueba de Kendall. Para todas las pruebas se usó como diferencia estadísticamente significativa un $p<0,05$. Se calcularon estos resultados usando el Software Stata 10.0 SE.

\section{Resultados}

Se registraron 31 pacientes operados de Pectus excatavum, todos tenían su ficha disponible y completa con los datos requeridos (tabla 1). De estos, 24 correspondían a varones (77\%) y 7 al sexo femenino (23\%). Veinticuatro pacientes aún tenían la barra al momento de la encuesta; sólo se había extraído a 7 pacientes, en los cuales había transcurrido el tiempo necesario para su retiro (entre 2 y 3 años).

La mediana de edad de los pacientes al momento de la cirugía fue de 12 (9-15) años. La mediana de edad del grupo al momento de la encuesta fue de 13 (10-16). La mediana de meses transcurrida desde la cirugía hasta el 
Tabla 1. Descripción de pacientes

\begin{tabular}{|c|c|}
\hline $\begin{array}{l}\text { Sexo } \\
\text { - Mujeres } \\
\text { - Hombres }\end{array}$ & $\begin{array}{r}7 / 31 \\
24 / 31\end{array}$ \\
\hline Edad al momento de cirugía & $12(9-15)$ años \\
\hline Edad al momento de entrevista & $13(10-16)$ años \\
\hline $\begin{array}{l}\text { Tiempo transcurrido entre cirugía } \\
\text { y entrevista }\end{array}$ & $\begin{array}{l}12 \text { meses } \\
\text { (promedio) }\end{array}$ \\
\hline $\begin{array}{l}\text { Causa de cirugía } \\
\text { - Funcional } \\
\text { - Estética }\end{array}$ & $\begin{array}{l}28 / 31 \\
15 / 31\end{array}$ \\
\hline Índice de Haller & $3,5(3-3,75)$ \\
\hline Ecocardiograma alterado* & $3 / 31$ \\
\hline $\begin{array}{l}\text { Espirometría normal } \\
\text { - Patrón obstructivo } \\
\text { - Patrón restrictivo }\end{array}$ & $\begin{array}{r}24 / 31 \\
3 / 31 \\
4 / 31\end{array}$ \\
\hline Tiempo quirúrgico & $1,6(1,5-2,25) \mathrm{H}$. \\
\hline Ingreso en algún momento a UCIP & $20 / 31$ \\
\hline Estadía hospitalaria & 4 (4-5) días \\
\hline $\begin{array}{l}\text { Complicaciones } \\
\text { - Infección de herida operatoria }\end{array}$ & $\begin{array}{l}5 / 31 \\
2 / 31\end{array}$ \\
\hline
\end{tabular}

momento de la entrevista fue de 12 (4-24) meses.

En 28 pacientes la indicación de cirugía fue por causa estética (sicológica) y funcional (intolerancia al ejercicio, molestias respiratorias, dolor torácico), algunos pacientes destacaron más la primera causa ${ }^{12}$, mientras en otros predominaron las molestias funcionales ${ }^{16}$. Sólo 3 pacientes reconocieron que el único motivo de su cirugía era estético.

\section{Evaluación preoperatoria}

TAC de tórax: disponible en la ficha clínica de 27 pacientes, índice de Haller con mediana de 3,5 (3-3,75).

Espirometría: a 30 pacientes se les realizó espirometría, 23 de ellos con espirometría normal, 4 con limitación ventilatoria restrictiva leve, 2 con limitación obstructiva moderada y 1 paciente con limitación ventilatoria mixta, obstructiva moderada y restrictiva leve.

El tiempo operatorio promedio fue de $1,6 \mathrm{~h}$ con un rango que varió desde 1,5 a $2,25 \mathrm{~h}$.
Tabla 2. Cuestionario de Nuss Modificado, según tabulación univariada de valor promedio obtenido en cada pregunta (1-3)

\begin{tabular}{|c|c|c|}
\hline Pregunta & Pre & Post \\
\hline ¿Cómo te sientes con tu aspecto? & 2,03 & 3,83 \\
\hline $\begin{array}{l}\text { ¿Cómo te sientes con el aspecto de tu tórax al } \\
\text { mirarte al espejo sin camisa? }\end{array}$ & 1,67 & 3,83 \\
\hline $\begin{array}{l}\text { ¿Cómo te sientes por lo que dice la gente de tu } \\
\text { tórax al mirarte? }\end{array}$ & 2,32 & 3,70 \\
\hline
\end{tabular}

Cuestionario de Nuss: Se evaluaron 31 pacientes mediante cuestionario de Nuss y de satisfacción postoperatoria. Los resultados se muestran en las tablas 1 y 2 .

\section{Respuestas 1-3:}

$4=$ Muy feliz.

$3=$ Más o menos feliz.

$2=$ Más o menos infeliz.

$1=$ Infeliz.

Veinte pacientes hicieron el postoperatorio en UCIP, con una estadía promedio de 2,31 días. Uno de ellos debió reingresar debido a una complicación pulmonar (atelectasia).

Se utilizó en todos los pacientes analgesia inicial por catéter peridural y posteriormente analgesia endovenosa en base a dipirona, ketorolaco y tramadol, ajustadas las dosis según peso.

Cuatro pacientes $(12,9 \%)$ presentaron complicaciones, las cuales fueron: 2 pacientes con infección de la herida operatoria, 1 paciente con atelectasia del lóbulo superior derecho, el cual requirió reingreso a UCIP y 1 paciente con un Síndrome de Claude Bernard Horner transitorio, atribuible al bloqueo con anestesia peridural. En la totalidad de los pacientes se utilizó 1 barra. En 3 pacientes se utilizó 2 estabilizadores (a consideración del cirujano, para tener mayor seguridad con respecto a la estabilidad de la barra), en el resto (28 pacientes) se utilizó sólo 1 estabilizador, ubicado en el lado izquierdo del tórax, más fijación al lado derecho con puntos pericostales de PDS o Vicryl.

El tiempo de estadía hospitalaria correspondió a 4 días, con un rango entre 4 y 5 días.

Los resultados respecto a la satisfacción post quirúrgica según el cuestionario de Nuss para pacientes pediátricos ${ }^{14}$ se muestran en las tablas $2 \mathrm{y}$ 3.

En las tablas 4 y 5 se encuentran las respuestas al cuestionario de Krasopoulos ${ }^{3}$, el cual también permite evaluar el grado de satisfacción que tienen los pacientes, con el procedimiento al cual fueron sometidos. 
Tabla 3. Cuestionario de Nuss Modificado, según tabulación univariada de valor promedio obtenido en cada pregunta (4-12)

\begin{tabular}{|c|c|c|}
\hline Pregunta & Pre & Post \\
\hline ¿La gente se ríe de ti por el aspecto de tu tórax? & 2,49 & 3,61 \\
\hline $\begin{array}{l}\text { ¿El aspecto del tórax te lleva a evitar hacer cosas } \\
\text { delantede desconocidos, compañeros o amigos? }\end{array}$ & 2,03 & 3,65 \\
\hline ¿Ocultas tu tórax? & 2,19 & 3,84 \\
\hline $\begin{array}{l}\text { ¿Te has sentido incomodo de cómo la gente } \\
\text { mira tu tórax? }\end{array}$ & 2,48 & 3,97 \\
\hline $\begin{array}{l}\text { ¿Te sientes avergonzado cuando la gente mira } \\
\text { tu tórax? }\end{array}$ & 2,65 & 3,90 \\
\hline ¿Te sientes mal por tu enfermedad? & 1,74 & 3,87 \\
\hline ¿Tienes apuro por terminar las clases de gimnasia? & 2,58 & 3,81 \\
\hline ¿Sientes que te cuesta respirar por tu tórax? & 1,77 & 3,55 \\
\hline ¿Te cansas rápidamente al hacer ejercicio? & 1,74 & 3,65 \\
\hline
\end{tabular}

Respuestas 4-12:

$1=$ Frecuentemente.

$2=A$ veces

$3=$ Casi nunca.

$4=$ Nunca.

\section{Discusión}

Respecto a la distribución por sexo, en nuestro estudio, como en los de otros autores, la cantidad de pacientes de sexo femenino fue baja o nula, lo que concuerda con la predominancia descrita en varones para la enfermedad ${ }^{1,3,14,15}$.

El índice de Haller se considera normal hasta $3,25^{17}$, el promedio de nuestros pacientes de 3,5, es algo inferior a lo publicado por Nuss y Varela en sus series ${ }^{15,18}$ pero similar a lo publicado en otra serie nacional ${ }^{19}$.

En la práctica diaria vemos que cada vez un mayor número de pacientes, con defectos que catalogaríamos como moderados (con índices de Haller un poco sobre lo normal), buscan ayuda mediante esta técnica quirúrgica para corregir su defecto, en vista de los buenos resultados publicados en diferentes medios y que han trascendido

Tabla 4. Cuestionario de satisfacción post quirúrgica

\begin{tabular}{|c|c|c|c|}
\hline Preguntas & & Respuestas & \\
\hline $\begin{array}{l}\text { Cómo te sientes en general de salud, después } \\
\text { de la operación }\end{array}$ & $\begin{array}{c}\text { Mejor } \\
\text { (28 pacientes - 90,31\%) }\end{array}$ & $\begin{array}{c}\text { Igual } \\
\text { (2 pacientes }-6,45 \%)\end{array}$ & $\begin{array}{c}\text { Peor } \\
\text { (1 paciente - 3,22\%) }\end{array}$ \\
\hline $\begin{array}{l}\text { Cómo está tu capacidad para hacer educación } \\
\text { física y ejercicio después de la cirugía }\end{array}$ & $\begin{array}{c}\text { Mejor } \\
\text { (26 pacientes }-83,86 \%)\end{array}$ & $\begin{array}{c}\text { Igual } \\
\text { (3 pacientes }-9,67 \%)\end{array}$ & $\begin{array}{c}\text { Peor } \\
\text { (2 pacientes }-6,45 \%)\end{array}$ \\
\hline $\begin{array}{l}\text { En qué grado el aspecto de tu tórax interfería } \\
\text { en tus actividades sociales antes de la cirugía }\end{array}$ & $\begin{array}{c}\text { Mucho } \\
\text { (8 pacientes }-25,8 \%)\end{array}$ & $\begin{array}{c}\text { Moderado } \\
\text { (3 pacientes }-9,67 \%)\end{array}$ & $\begin{array}{c}\text { Poco } \\
\text { (20 pacientes }-64,5 \%)\end{array}$ \\
\hline $\begin{array}{l}\text { En qué grado el aspecto de tu tórax interfiere } \\
\text { en tus actividades sociales después de la cirugía }\end{array}$ & $\begin{array}{c}\text { Casi nada } \\
(25 \text { pacientes }-80,64 \%)\end{array}$ & $\begin{array}{c}\text { Poco } \\
(5 \text { pacientes }-16,12 \%)\end{array}$ & $\begin{array}{c}\text { Mucho } \\
\text { (1 paciente }-3,22 \%)\end{array}$ \\
\hline $\begin{array}{l}\text { Satisfacción con el aspecto postoperatorio } \\
\text { total }\end{array}$ & $\begin{array}{l}\text { Extremadamente satisfecho } \\
(24 \text { pacientes }-77,41 \%)\end{array}$ & $\begin{array}{c}\text { Muy satisfecho } \\
\text { (5 pacientes }-16,12 \%)\end{array}$ & $\begin{array}{c}\text { Satisfecho } \\
\text { (2 pacientes }-6,45 \%)\end{array}$ \\
\hline $\begin{array}{l}\text { Te sientes incómodo con las cicatrices de la } \\
\text { cirugía }\end{array}$ & $\begin{array}{c}\text { No } \\
\text { (25 pacientes }-80,64 \%)\end{array}$ & $\begin{array}{c}\text { Poco } \\
\text { (5 pacientes }-16,12 \%)\end{array}$ & $\begin{array}{c}\text { Mucho } \\
\text { (1 paciente - 3,22\%) }\end{array}$ \\
\hline $\begin{array}{l}\text { Cuánto impacto tuvo la cirugía en tu vida } \\
\text { social }\end{array}$ & $\begin{array}{c}\text { Casi nada } \\
\text { (6 pacientes }-19,35 \%)\end{array}$ & $\begin{array}{c}\text { Poco } \\
\text { (10 pacientes }-32,25 \%)\end{array}$ & $\begin{array}{c}\text { Mucho } \\
\text { (15 pacientes }-48,38 \%)\end{array}$ \\
\hline Está consciente que tiene una barra metálica & $\begin{array}{c}\text { Mucho } \\
\text { (15 pacientes }-48,38 \%)\end{array}$ & $\begin{array}{c}\text { Poco } \\
\text { (15 pacientes }-48,38 \%)\end{array}$ & $\begin{array}{c}\text { Casi nada } \\
\text { (1 paciente }-3,22 \%)\end{array}$ \\
\hline $\begin{array}{l}\text { Cuál es la satisfacción del resultado final de } \\
\text { la cirugía }\end{array}$ & $\begin{array}{l}\text { Extremadamente satisfecho } \\
(27 \text { pacientes }-87,09 \%)\end{array}$ & $\begin{array}{c}\text { Satisfecho } \\
\text { (3 pacientes }-9,67 \%)\end{array}$ & $\begin{array}{c}\text { Disconforme } \\
\text { (1 paciente }-3,22 \%)\end{array}$ \\
\hline Encuentra que el pecho se ve diferente & $\begin{array}{c}\text { Mucho } \\
\text { (29 pacientes - 93,54\%) }\end{array}$ & $\begin{array}{c}\text { Poco } \\
\text { (1 paciente }-3,22 \%)\end{array}$ & $\begin{array}{c}\text { Casi nada } \\
\text { (1 paciente }-3,22 \%)\end{array}$ \\
\hline $\begin{array}{l}\text { Si pudiera retroceder el tiempo, se realizaría } \\
\text { de nuevo la cirugía }\end{array}$ & $\begin{array}{c}\text { Sí } \\
\text { (30 pacientes }-96,77 \%)\end{array}$ & $\begin{array}{c}\text { No } \\
\text { (1 paciente }-3,22 \%)\end{array}$ & \\
\hline
\end{tabular}

Cuestionario de satisfacción post quirúrgica. Todos los pacientes contestaron el cuestionario, administrado telefónicamente. Los resultados se muestran en las siguientes tablas (tablas 4 y 5 ). 
Tabla 5. Autoestima pre y postoperatoria

\begin{tabular}{|c|c|c|c|}
\hline Pregunta & $\begin{array}{l}\text { Pre intervención } \\
\text { mediana (RIC) }\end{array}$ & $\begin{array}{l}\text { Post intervención } \\
\text { mediana (RIC) }\end{array}$ & $\mathbf{p}$ \\
\hline ¿Cómo te sientes con tu aspecto? & $2(1-3)$ & $4(4-4)$ & $<0,001$ \\
\hline ¿Cómo te sientes con el aspecto de tu tórax al mirarte al espejo sin camisa? & $2(1-2)$ & $4(4-4)$ & $<0,001$ \\
\hline ¿Cómo te sientes por lo que dice la gente de tu tórax al mirarte? & $2(2-3)$ & $4(4-4)$ & $<0,001$ \\
\hline ¿La gente se ríe de ti por el aspecto de tu tórax? & $2(1-4)$ & $4(3-4)$ & $<0,001$ \\
\hline $\begin{array}{l}\text { ¿El aspecto del tórax te lleva a evitar hacer cosas delante de desconocidos, } \\
\text { compañeros o amigos? }\end{array}$ & $2(1-4)$ & $4(4-4)$ & $<0,001$ \\
\hline ¿Ocultas tu tórax? & $2(1-4)$ & $4(4-4)$ & $<0,001$ \\
\hline ¿Te has sentido incomodo de cómo la gente mira tu tórax? & $2(1-4)$ & $4(4-4)$ & $<0,001$ \\
\hline ¿Te sientes avergonzado cuando la gente mira tu tórax? & $2(2-4)$ & $4(4-4)$ & $<0,001$ \\
\hline ¿Te sientes mal por tu enfermedad? & $2(1-2)$ & $4(4-4)$ & $<0,001$ \\
\hline ¿Tienes apuro por terminar las clases de gimnasia? & $3(1-4)$ & $4(4-4)$ & $<0,001$ \\
\hline ¿Sientes que te cuesta respirar por tu tórax? & $2(1-2)$ & $4(3-4)$ & $<0,001$ \\
\hline ¿Te cansas rápidamente al hacer ejercicio? & $1(1-2)$ & $4(3-4)$ & $<0,001$ \\
\hline
\end{tabular}

al público no médico. No cabe duda que al ser la principal indicación quirúrgica de tipo estética, la percepción del defecto por parte del paciente es diferente a la del equipo médico.

Se ha relacionado el Pectus excatavum con una mayor incidencia de patología respiratoria, pero esto no se vio mayormente reflejado en la espirometría de nuestros pacientes $(77 \%$ con espirometría normal).

Aun así, la mayoría de los niños operados relatan una clara mejoría en su estado físico y en su capacidad para practicar deportes, transcurrido un tiempo después de su cirugía.

La técnica quirúrgica empleada en nuestro estudio es similar a lo descrito en estudios internacionales, como los de Nuss, Krasopoulus y otros ${ }^{3,14,15}$. Los resultados obtenidos en relación al tiempo operatorio, estadía hospitalaria y complicaciones son concordantes con cifras nacionales e internacionales, publicadas para el tratamiento, tanto de pacientes adultos como niños ${ }^{11,15,18,19}$. Esto confirma que es una técnica reproducible y segura para nuestros pacientes.

En la gran mayoría de los casos, la causa de cirugía fue mixta (tanto estética como funcional) y prácticamente todos nuestros pacientes tenían trastornos psicológicos secundarios a la deformidad de la pared torácica, con gran repercusión en su autoestima.
El cuestionario de Nuss evalúa en primer lugar el grado de felicidad del paciente respecto al aspecto de su tórax, en nuestra encuesta, se aprecia que existe una diferencia estadísticamente significativa, en la opinión de los pacientes antes y después de la cirugía. Este alto grado de satisfacción postquirúrgica, con un $100 \%$ de los pacientes que declara sentirse "muy feliz" o "más o menos feliz" (tabla 2), es independiente de los factores que revelan el grado de la lesión (índice de Haller) o factores propios de los pacientes (edad, sexo).

Esto se demuestra al cruzar distintas variables como sexo, edad actual, edad a la cirugía, tiempo trascurrido desde la encuesta, fecha de la cirugía e índice de Haller, con los resultados de satisfacción postquirúrgica. Los resultados revelan que no hay diferencias estadísticamente significativas, lo que es consistente con que el factor principal que determina el cambio entre pre y post, es exclusivamente la cirugía.

Llama la atención que la mayoría de los niños entrevistados son tímidos y retraídos, esto debido tanto a la malformación misma, como a los comentarios y miradas del entorno. En general se abstienen de participar en actividades en las cuales el tórax es necesariamente expuesto, como ocurre en la natación, ejercicio y educación física. Al corregir la deformidad 
existe una marcada mejoría de la autoestima, de los síntomas respiratorios y cardíacos y de la percepción que tienen del entorno hacia ellos.

La autoestima preoperatoria medida en una escala de 1 (más baja) a 10 (más alta), mejora después de la cirugía, de 6 a 9, con significancia estadística. Este objetivo principal se consigue ampliamente con el procedimiento de Nuss y es concordante con lo publicado por él, respecto a los resultados de la cirugía ${ }^{19}$.

El dolor postoperatorio, que aparece como una de las grandes limitantes de la cirugía y que fue descrito como importante por el $52 \%$ de los pacientes, prácticamente desaparece algún tiempo después y en general no interfiere en las actividades cotidianas $88 \%$ ).

Creemos que las principales conclusiones, son las que se deducen de las respuestas de los pacientes al preguntarles: si se sienten mal con su enfermedad (tabla 2, pregunta 9), con un dramático cambio de puntaje post cirugía de 1,74 (a veces o frecuentemente) a 3,87 (nunca) y al preguntarles su satisfacción con el aspecto postoperatorio total (tabla 4, pregunta 5), en que todos los pacientes se declaran, en mayor o menor grado, satisfechos con el aspecto postoperatorio $(77,4 \%$ se declara extremadamente satisfecho, el 16,1\% muy satisfecho y el 6,5\% satisfecho). Esta respuesta varía levemente en la pregunta 9 sobre satisfacción con el resultado final de la cirugía en que 30 pacientes se declaran extremadamente satisfechos o satisfechos y sólo 1 disconforme.

Más aun, ante la pregunta sobre si se operarían de nuevo, 30 de 31 pacientes responde afirmativamente (tabla 4, pregunta 12).

La tabla 5 muestra una diferencia estadísticamente significativa en todas las variables encuestadas, sobre todo en relación a como se sienten con su aspecto y si se sienten avergonzados por su malformación.

Los pacientes se declaran claramente beneficiados y felices con los resultados de la cirugía, independientemente de la apreciación subjetiva que los médicos u otros observadores puedan tener sobre el procedimiento, en concordancia con lo publicado en la literatura ${ }^{16}$.

En definitiva, la corrección del Pectus excatavum mediante técnica de Nuss demuestra un efecto positivo, tanto en la esfera física, como biopsicosocial de los pacientes, al corregir su deformidad, lo que se ve claramente reflejado en una mejora de la autoestima y en la percepción de su estado de salud.

Anexo 1. Variables epidemiológicas estudio Pectum exacavatum

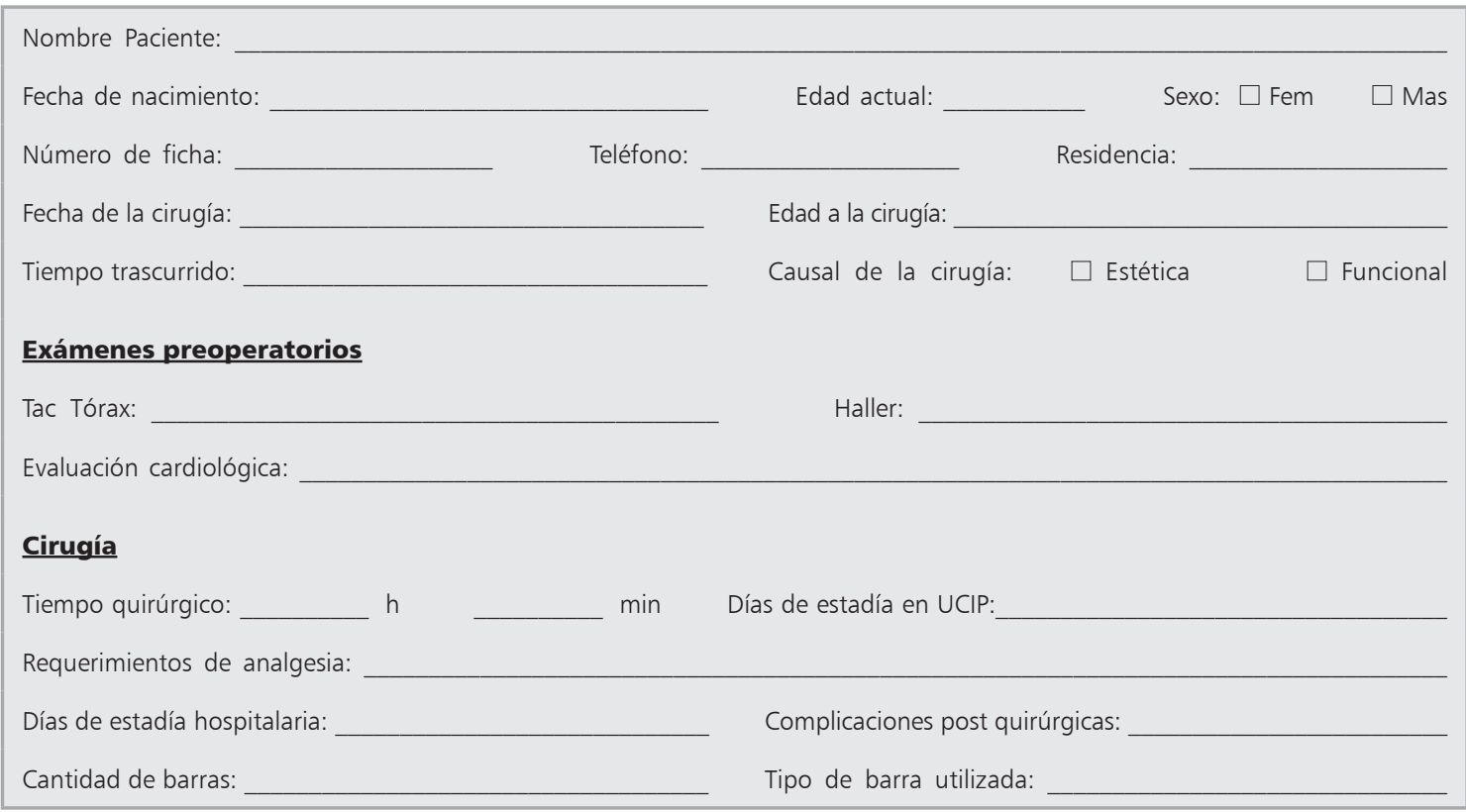


KRAL A. y cols.

\section{Anexo 2. Cuestionario de Nuss Pediátrico}

\begin{tabular}{|c|c|c|c|c|}
\hline \multicolumn{4}{|c|}{ 1.- Cómo te sientes con tu aspecto general: } & \\
\hline Precirugía: & $\square$ muy feliz & $\square$ más o menos feliz & $\square$ más o menos infeliz & $\square$ infeliz \\
\hline Post cirugía: & $\square$ muy feliz & $\square$ más o menos feliz & $\square$ más o menos infeliz & $\square$ infeliz \\
\hline \multicolumn{5}{|c|}{ 2.- Cómo te sientes con el aspecto de tu tórax al mirarte al espejo sin camisa } \\
\hline Precirugía: & $\square$ muy feliz & $\square$ más o menos feliz & $\square$ más o menos infeliz & $\square$ infeliz \\
\hline Post cirugía: & $\square$ muy feliz & $\square$ más o menos feliz & $\square$ más o menos infeliz & $\square$ infeliz \\
\hline \multicolumn{5}{|c|}{ 3.- Como te sientes por lo que dice la gente de tu tórax al mirarte. } \\
\hline Pre cirugía: & $\square$ muy feliz & $\square$ más o menos feliz & $\square$ más o menos infeliz & $\square$ infeliz \\
\hline Post cirugía: & $\square$ muy feliz & $\square$ más o menos feliz & $\square$ más o menos infeliz & $\square$ infeliz \\
\hline \multicolumn{5}{|c|}{ 4.- La gente se ríe de ti por el aspecto de tu tórax: } \\
\hline Pre cirugía: & $\square$ frecuentemente & $\square$ a veces & $\square$ casi nunca & $\square$ nunca \\
\hline Post cirugía: & $\square$ frecuentemente & $\square$ a veces & $\square$ casi nunca & $\square$ nunca \\
\hline
\end{tabular}

5.- El aspecto de tu tórax te lleva a evitar hacer cosas delante de desconocidos, compañeros o amigos?:

Pre cirugía:

$\square$ frecuentemente

$\square$ a veces

$\square$ casi nunca

$\square$ nunca

Post cirugía:

$\square$ frecuentemente

$\square$ a veces

$\square$ casi nunca

$\square$ nunca

6.- Ocultas tu tórax

Pre cirugía:

$\square$ frecuentemente

$\square$ a veces

$\square$ casi nunca

$\square$ nunca

Post cirugía:

$\square$ frecuentemente

$\square$ a veces

$\square$ casi nunca

$\square$ nunca

7.- Te has sentido incómodo de cómo la gente mira tu tórax:

\begin{tabular}{|c|c|c|c|c|}
\hline Pre cirugía: & $\square$ frecuentemente & $\square$ a veces & $\square$ casi nunca & $\square$ nunca \\
\hline Post cirugía: & $\square$ frecuentemente & $\square$ a veces & $\square$ casi nunca & $\square$ nunca \\
\hline \multicolumn{5}{|c|}{ 8.- Te sientes avergonzado cuando la gente mira tu tórax: } \\
\hline Pre cirugía: & $\square$ frecuentemente & $\square$ a veces & $\square$ casi nunca & $\square$ nunca \\
\hline Post cirugía: & $\square$ frecuentemente & $\square$ a veces & $\square$ casi nunca & $\square$ nunca \\
\hline \multicolumn{5}{|c|}{ 9.- Te sientes mal por tu enfermedad: } \\
\hline Pre cirugía: & $\square$ frecuentemente & $\square$ a veces & $\square$ casi nunca & $\square$ nunca \\
\hline Post cirugía: & $\square$ frecuentemente & $\square$ a veces & $\square$ casi nunca & $\square$ nunca \\
\hline \multicolumn{5}{|c|}{ 10.- Tienes apuro por terminar las clases de gimnasia: } \\
\hline Pre cirugía: & $\square$ frecuentemente & $\square$ a veces & $\square$ casi nunca & $\square$ nunca \\
\hline Post cirugía: & $\square$ frecuentemente & $\square$ a veces & $\square$ casi nunca & $\square$ nunca \\
\hline \multicolumn{5}{|c|}{ 11.- Sientes que te cuesta respirar por tu tórax: } \\
\hline Pre cirugía: & $\square$ frecuentemente & $\square$ a veces & $\square$ casi nunca & $\square$ nunca \\
\hline Post cirugía: & $\square$ frecuentemente & $\square$ a veces & $\square$ casi nunca & $\square$ nunca \\
\hline \multicolumn{5}{|c|}{ 12.-Tu tórax te lleva a cansarte más de lo habitual: } \\
\hline Pre cirugía: & $\square$ frecuentemente & $\square$ a veces & $\square$ casi nunca & $\square$ nunca \\
\hline Post cirugía: & $\square$ frecuentemente & $\square$ a veces & $\square$ casi nunca & $\square$ nunca \\
\hline
\end{tabular}




\section{Anexo 3. Satisfacción post quirúrgica}

Contesta las preguntas de acuerdo cómo te sientes (o se siente tu hijo) después de la cirugía.

1. Cómo te sientes en general de salud, después de la operación:
$\square$ Mucho mejor
$\square$ Algo mejor
$\square$ Casi igual
$\square$ Algo peor ahora
$\square$ Mucho peor

2. Cómo está tu capacidad para hacer educación física y ejercicio después de la cirugía.
$\square$ Mucho mejor
$\square$ Algo mejor
$\square$ Casi igual
$\square$ Algo peor ahora
Mucho peor

3. En qué grado el aspecto de tu tórax interfería en tus actividades sociales antes de la cirugía:
$\square$ Demasiado
$\square$ Harto
$\square$ Moderado
$\square$ Un poco
$\square$ Casi nada

4. En qué grado el aspecto de tu tórax interfiere en tus actividades sociales después de la cirugía:
$\square$ Demasiado
$\square$ Harto
$\square$ Moderado
$\square$ Un poco
$\square$ Casi nada

5. Satisfacción con el aspecto postoperatorio total

$\square$ Extremadamente satisfecho $\quad \square$ Muy satisfecho $\quad \square$ Satisfecho $\quad \square$ Desconforme $\quad \square$ Muy desconforme

6. Te sientes incómodo con las cicatrices de la cirugía:
$\square$ No
$\square$ Un poquito
$\square$ Más o menos
$\square$ Mucho
Muchísimo

7. Cuánto impacto tuvo la cirugía en tu vida social
$\square$ Demasiado
$\square$ Harto
$\square$ Moderado
$\square$ Un poco
$\square$ Casi nada

8. De uno a diez qué puntaje tenía de autoestima Pre operatoria:

9. De uno a diez qué puntaje tiene de autoestima Post operatoria:

10. Cuánto dolor tuvo durante su estadía hospitalaria:
$\square$ Demasiado
$\square$ Harto
$\square$ Moderado
$\square$ Un poco
$\square$ Casi nada

11. El dolor interfiere con sus actividades cotidianas:
$\square$ Demasiado
$\square$ Harto
$\square$ Moderado
$\square$ Un poco
$\square$ Casi nada

12. Siente dolor ahora:
$\square$ Demasiado
$\square$ Harto
$\square$ Moderado
$\square$ Un poco
$\square$ Casi nada

13. Está consciente que tiene una barra metálica:
$\square$ Demasiado
$\square$ Harto
$\square$ Moderado
$\square$ Un poco
Casi nada

14. Cuál es la satisfacción del resultado final de la cirugía.
$\square$ Extremadamente satisfecho
$\square$ Muy satisfecho
$\square$ Satisfecho
$\square$ Desconforme
$\square$ Muy desconforme

15. Encuentra que el pecho se ve diferente:

$\square$ Demasiado $\quad \square$ Harto $\quad \square$ Moderado $\quad \square$ Un poco $\quad \square$ Casi nada

16. Si pudiera retroceder el tiempo, se realizaría de nuevo la cirugía: $\square$ Sí $\quad \square$ No

\section{Referencias}

1.- Goretsky M, Kelly R: Chest wall anomalies: Pectus excavatum and Pectus carinatum.

2.- Martínez C, Guevara L, Aguillon A: Presentación de un caso de corrección cosmética de Pectus excavatum. Rev. Inst Nal Enf. Resp Mex v. 18 n. 2 México abr-jun. 2005.

3.- Croitoru D, Nuss D, Krasopoulos G, Dusme M, Ladas $G$, Goldstraw P: Nuss procedure improves the quality of life in young male adults with Pectus excavatum deformity. European Journal of Cardio-thoracic Surgery Vol. 29 (2006) 1-5.

4.- Anzieta J, Arancibia V, Ramírez J, Venturelli F: Vídeotoracoscopia en el tratamiento del Pectus excavatum. Cuad Cir 2005; 19: 60-5.

5.- Guntheroth W, Spiers P: Cardiac Function Before and After Surgery for Pectus excavatum. Am J Cardiol 2007; 99: 1762-4. 
6.- Alonso J: La medida de la calidad de vida relacionada con la salud en la investigación y la práctica clínica. Gac Sanit 2000; 14 (2): 163-7.

7.- Vegunta R, Pacheco E, Wallace L: Complications associated with the Nuss procedure: continued evolution of the learning curve. American Journal of Surgery 2008; 195: 313-31.

8.- Goretsky M, Nelly R, Croitoru D, Nuss D: Chest wall anomalies: Pectus excavatum and Pectus carinatum. Adolesc Med 2004; 15: 455-71

9.- Fefferman N, Pinkney L: Imaging Evaluation of Chest Wall Disorders in Children. Radiol Clin N Am 2005; 43: 355-70.

10.- McGuigan R, Azarow K: Congenital Chest Wall Defects. Surg Clin N Am 2006; 86: 353-70.

11.- Kelly, et al: Prospective Multicenter Study of Surgical Correctionof Pectus excavatum: Design, Perioperative Complications, Pain, and Baseline Pulmonary Function Facilitated by Internet-Based Data Collection. J Am Coll Surg 2007; 205: 205-16.

12.- Rajmil L, Estrada MD, Herdman M, Serra-Sutton V, Alonso J: Calidad de vida relacionada con la salud (CVRS) en la infancia y la adolescencia: revisión de la bibliografía y de los instrumentos adaptados en España. Gac Sanit 2001; 15 (Suppl 4): 34-43.
13.- Pinto-Prades J: Calidad de vida y asignación de recursos sanitarios. Gac Sanit 2000; 14 (2): 168-74.

14.- Lawson ML, Cash TF, Akers R, et al: A pilot study of the impact of surgical repair on disease-specific quality of life among patients with Pectus excavatum. J Pediatr Surg 2003; 38: 916-8.

15.- Nuss D, Kelly R, Croitoru D, Katz M: A 10-year review of a minimally invasive technique for the correction of Pectus excavatum. J Pediatr Surg 1998; 33: 545-52.

16.- Krasopoulos G, Dusmet M, Ladas G, Goldstraw P: Nuss procedure improves the quality of life in young male adults with Pectus excavatum deformity. European Journal of Cardio-thoracic Surgery 2006; 29: 1-5.

17.- Haller JA, Kramer SS, Lietman SA: Use of CT scans in selection of patients for Pectus excavatum surgery: a preliminary report. J Pediatric Surg 1987; 22: 904-6.

18.- Varela P, Herrera O, Fielbaum O: Pectus excavatum. Tratamiento con técnica mínimamente invasiva. Rev Chil. Pediatr 2002; 73 (3): 263-9.

19.- Prats $R$, González R, Venturelli F, Lazo D, Santolaya $R$, Rodríguez $P$ : Corrección mínimamente invasiva (Operación de Nuss) del Pectus excavatum en pacientes adultos. Rev Med Chile 2009; 137: 1583-90.

20.- Nuss D. Reparación mínimamente invasiva del Pectus excavatum. Cir Pediatr 2002; 15: 1-2. 